Fortschritt-Berichte VDl

VDI

Reihe 12

Verkehrstechnik/ Fahrzeugtechnik

Nr. 807

\title{
39. Internationales
}

\section{Wiener Motorensymposium 26.-27. April 2018}

veranstaltet vom

Österreichischen Verein für Kraftfahrzeugtechnik (ÖVK) und vom Institut für Fahrzeugantriebe und Automobiltechnik der Technischen Universität Wien

\section{$39^{\text {th }}$ International}

Vienna Motor Symposium

26-27 April 2018

organized by

the Austrian Society of Automotive Engineers (ÖVK) and the Institute for Powertrains and Automotive Technology, Vienna University of Technology

zusammengestellt von / presented by Univ.-Prof. Dr. Bernhard Geringer Univ.-Prof. Dr. Hans Peter Lenz

in zwei Bänden / in two volumes 


\section{AVL}

\section{INNOVATIVE TECHNOLOGY}

SUBJECTIVE FEELINGS

Fuel your passion for driving

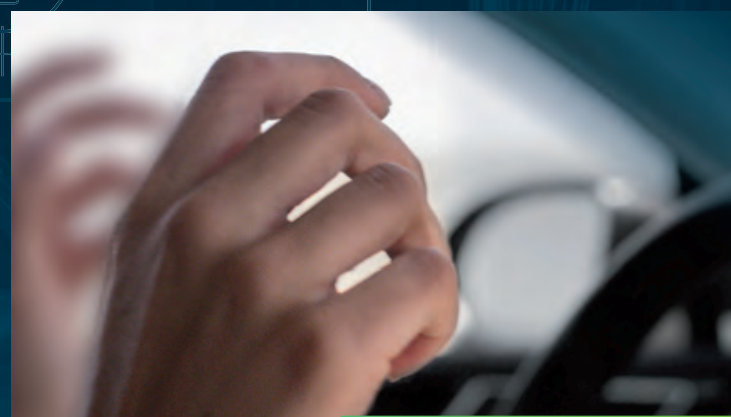

AUTONOMOUS DRIVING Free your mind to limitless possibilities

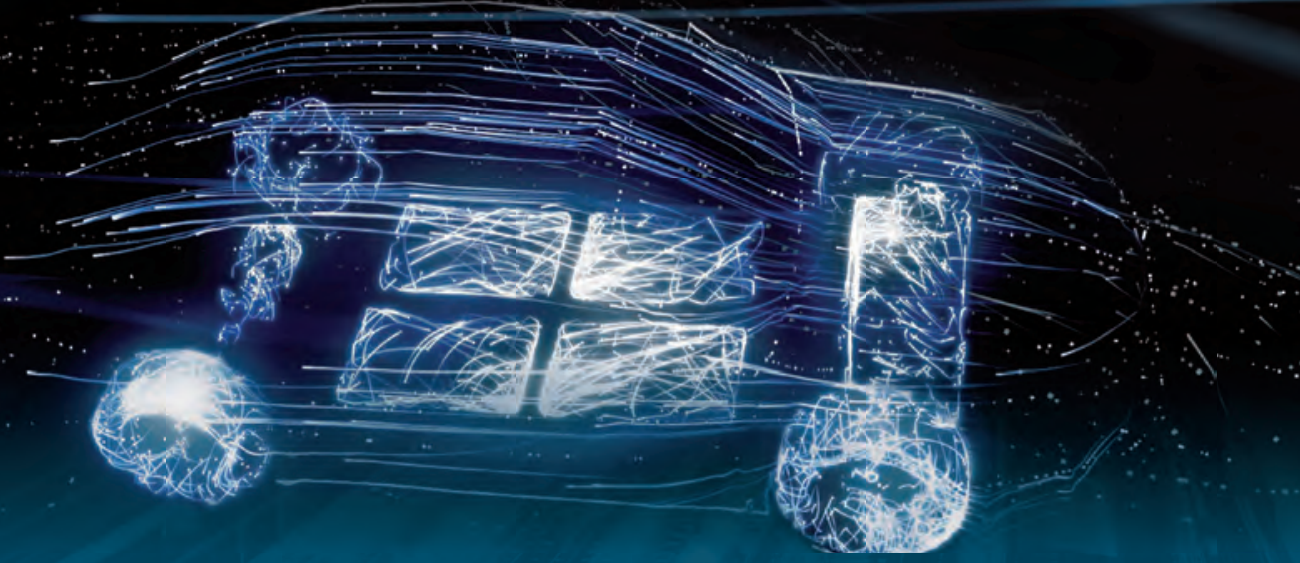




\section{Fortschritt-Berichte VDI}

\section{Reihe 12}

Verkehrstechnik/

Fahrzeugtechnik

\section{Internationales \\ Wiener Motorensymposium 26.-27. April 2018}

veranstaltet vom

Nr. 807

Österreichischen Verein für Kraftfahrzeugtechnik (ÖVK) und vom Institut für Fahrzeugantriebe und

Automobiltechnik der Technischen Universität Wien

$39^{\text {th }}$ International

Vienna Motor Symposium

26-27 April 2018

organized by

the Austrian Society of Automotive Engineers (ÖVK) and the Institute for Powertrains and

Automotive Technology, Vienna University of Technology

zusammengestellt von / presented by

Univ.-Prof. Dr. Bernhard Geringer

Univ.-Prof. Dr. Hans Peter Lenz

in zwei Bänden / in two volumes 
Geringer, Bernhard u. Lenz, Hans Peter (Hrsg.)

\section{Internationales Wiener Motorensymposium 26.-27. April 2018 in zwei Bänden. Band 1: erster Tag; Band 2: zweiter Tag 39th International Vienna Motor Symposium 26-27 April 2018 in two volumes. Volume 1: first day; Volume 2: second day}

Fortschr.-Ber. VDI Reihe 12 Nr. 807. Düsseldorf: VDI-Verlag 2018.

758 Seiten, 669 Bilder, 56 Tabellen.

ISBN 978-3-18-380712-3, ISSN 0178-9449, € 220,00/VDI-Mitgliederpreis € 198,00.

Für die Dokumentation: Neue Otto- und Dieselmotoren - Kraftstoffe der Zukunft - Neue Antriebe und Getriebe für LKW - Neue Brennverfahren / Motorsteverung - Elektrische Antriebe / Hybride Innovationen bei der Abgasreinigung - Brennstoffzellen - Blick in die Zukunft

Dieser zweibändige Bericht macht die Vorträge des 39. Internationalen Wiener Motorensymposiums (26./27. April 2018) einer breiten Öffentlichkeit zugänglich.

Ziel der Wiener Motorensymposien ist es, Themen von besonderer Aktualität auf dem Gebiet des Verbrennungsmotors zu behandeln. Das Werk befasst sich u. a. mit den oben angeführten Themen. Der USB-Stick enthält die Vorträge sowohl in der Originalversion als auch auf Englisch. Herausgeber: Univ.-Prof. Dr. techn. Dipl.-Ing. Bernhard Geringer, Vorsitzender des Österreichischen Vereins für Krafffahrzeugtechnik (ÖVK), Wien, und Univ.-Prof. Dr. techn. Dipl.-Ing. Hans Peter Lenz, Ehrenvorsitzender des Osterreichischen Vereins für Krafffahrzeugtechnik (ÖVK), Wien.

Keywords: New Otto and Diesel Engines - Future Fuels - New Powertrains and Transmissions for Heavy Duty Vehicles - New Combustion Processes / Engine Control - Electric Drives / Hybrids - Innovative Systems of Emission Control - Fuel Cells - View to the Future

Abstract: This two-volume report makes the presentations of the $39^{\text {th }}$ International Vienna Motor Symposium (April 26-27, 2018) available to a wider audience.

The objective of the Vienna Motor Symposia is to examine current topics of particular interest. Among others, the work examines the above mentioned topics. The USB flash drive contains the presentations in their original form and in English translation.

Edited by Univ.-Prof. Dr. techn. Dipl.-Ing. Bernhard Geringer, President of the ÖVK (Austrian Society of Automotive Engineers, Vienna) and Univ.-Prof. Dr. techn. Dipl.-Ing. Hans Peter Lenz, Honorary President of the OVK (Austrian Society of Automotive Engineers, Vienna).

\section{Bibliographische Information der Deutschen Bibliothek}

Die Deutsche Bibliothek verzeichnet diese Publikation in der Deutschen Nationalbibliographie; detaillierte bibliographische Daten sind im Internet unter www.dnb.de abrufbar.

\section{Bibliographic information published by the Deutsche Bibliothek}

(German National Library)

The Deutsche Bibliothek lists this publication in the Deutsche Nationalbibliographie

(German National Bibliography); detailed bibliographic data is available via internet at www.dnb.de.

\section{(c) VDI Verlag GmbH · Düsseldorf 2018}

Alle Rechte, auch das des auszugsweisen Nachdruckes, der auszugsweisen oder vollständigen Wiedergabe (Fotokopie, Mikrokopie), der Speicherung in Datenverarbeitungsanlagen, im Internet und das der Übersetzung, vorbehalten.

Als Manuskript gedruckt. Printed in Germany.

ISSN 0178-9449

ISBN 978-3-18-380712-3 


\section{VORWORT}

Die beiden vorliegenden Bände geben die Vorträge, die anlässlich des

39. Internationalen Wiener Motorensymposiums am 26. - 27. April 2018 gehalten werden, wieder. Der neueste Stand der Motoren- und Antriebsentwicklung wird damit von herausragenden Vortragenden präsentiert.

Allen Vortragenden und den sie entsendenden Unternehmen bzw. Institutionen sei auch an dieser Stelle für ihre Beiträge herzlichst gedankt.

Alle Vorträge sind auf dem den Symposiumsunterlagen beigefügten USB-Stick sowohl in der Originalversion als auch in Übersetzung (Englisch bzw. Deutsch) enthalten.

Die „Literatursuche“ auf der Homepage des Internationalen Wiener Motorensymposiums (www.wiener-motorensymposium.at) bietet die Möglichkeit, mit Hilfe von Suchbegriffen die Vortragstitel, Autoren und Firmen der vorangegangenen Motorensymposien seit 1981 (Englisch ab 1999) zu finden. Die Liste der Publikationen (Sammelbände der Vorträge der Internationalen Wiener Motorensymposien) 1979 - 2018, die auch die breitere Öffentlichkeit über die Inhalte der Symposien informieren sollen, finden Sie auf der folgenden Seite.

Das 40. Internationale Wiener Motorensymposium findet am 16. - 17. Mai 2019 statt, wozu schon jetzt herzlich eingeladen wird.

Univ.-Prof. Dr. Bernhard Geringer Vorsitzender des Österreichischen Vereins für Kraftfahrzeugtechnik (ÖVK)
Univ.-Prof. Dr. Hans Peter Lenz Ehrenvorsitzender des Österreichischen Vereins für Kraftfahrzeugtechnik (ÖVK)

\section{FOREWORD}

These two volumes contain all the lectures given at the $39^{\text {th }}$ International Vienna Motor Symposium, which took place on $26^{\text {th }}$ and $27^{\text {th }}$ April, 2018. In these lectures, outstanding automotive engineers presented various aspects of state-of-the-art of engine and powertrain technology.

At this point, we would like to express our heartfelt thanks to all lecturers, as well as the companies and institutions they represent, for their contributions to our conference.

The original versions as well as the translations (German resp. English language) of all lectures are available on the USB flash drive which is attached to the symposium documents.

The word-search facility at www.vienna-motorsymposium.com - the website of the International Vienna Motor Symposium - allows direct access to the titles of lectures, together with information on all the authors and companies that have participated in the International Vienna Motor Symposia since 1999 (in German since 1981).

The publications list - which is to be found on the following page - provides an anthology of all lectures presented at the International Vienna Motor Symposia between 1979 and 
2018. It is intended to provide a broader public visibility with additional information on the topics and subject matter dealt with by these conferences.

The $40^{\text {th }}$ International Vienna Motor Symposium is to take place on the $16^{\text {th }}$ and $17^{\text {th }}$ May, 2019 , and may we take this early opportunity to extend you an invitation.

Univ.-Prof. Dr. Bernhard Geringer President of ÖVK

(Austrian Society of Automotive Engineers)
Univ.-Prof. Dr. Hans Peter Lenz

Honorary President of ÖVK

(Austrian Society of Automotive Engineers) 


\section{INTERNATIONALE WIENER MOTORENSYMPOSIEN Liste der Publikationen:}

"1.Internationales Wiener Motorensymposium“, ÖIZ, 124.Jahrgang (1979)

„2.Internationales Wiener Motorensymposium", VDI-Fortschritt Berichte, Reihe 6, Nr.69 (1980)

„3.Internationales Wiener Motorensymposium“, VDI-Fortschritt Berichte, Reihe 6, Nr.81 (1981)

"4.Internationales Wiener Motorensymposium“, VDI-Fortschritt Berichte, Reihe 6, Nr.103 (1982)

„5.Internationales Wiener Motorensymposium“, VDI-Fortschritt Berichte, Reihe 6, Nr.127 (1983)

„6. Internationales Wiener Motorensymposium“, VDI-Fortschritt Berichte, Reihe 6, Nr.173 (1985)

"7.Internationales Wiener Motorensymposium“, VDI-Fortschritt Berichte, Reihe 12, Nr.74 (1986)

„8.Internationales Wiener Motorensymposium“, VDI-Fortschritt Berichte, Reihe 12, Nr.86 (1987)

„9.Internationales Wiener Motorensymposium“, VDI-Fortschritt Berichte, Reihe 12, Nr.99 (1988)

„10. Internationales Wiener Motorensymposium“, VDI-Fortschritt Berichte, Reihe 12, Nr.122 (1989)

"11.Internationales Wiener Motorensymposium", VDI-Fortschritt Berichte, Reihe 12, Nr.141 (1990)

"12. Internationales Wiener Motorensymposium", VDI-Fortschritt Berichte, Reihe 12, Nr.150 (1991)

"13.Internationales Wiener Motorensymposium“, VDI-Fortschritt Berichte, Reihe 12, Nr.167 (1992)

„14.Internationales Wiener Motorensymposium“, VDI-Fortschritt Berichte, Reihe 12, Nr.182 (1993)

"15. Internationales Wiener Motorensymposium“, VDI-Fortschritt Berichte, Reihe 12, Nr.205 (1994)

"16.Internationales Wiener Motorensymposium“, VDI-Fortschritt Berichte, Reihe 12, Nr.239 (1995)

"17.Internationales Wiener Motorensymposium“, VDI-Fortschritt Berichte, Reihe 12, Nr.267 (1996)

"18. Internationales Wiener Motorensymposium", VDI-Fortschritt Berichte, Reihe 12, Nr.306 (1997)

"19.Internationales Wiener Motorensymposium“, VDI-Fortschritt Berichte, Reihe 12, Nr.348 (1998)
"20.Internationales Wiener Motorensymposium“, VDI-Fortschritt Berichte, Reihe 12, Nr.376 (1999)

„21. Internationales Wiener Motorensymposium“, VDI-Fortschritt Berichte, Reihe 12, Nr.420 (2000)

„22.Internationales Wiener Motorensymposium", VDI-Fortschritt Berichte, Reihe 12, Nr.455 (2001)

"23.Internationales Wiener Motorensymposium", VDI-Fortschritt Berichte, Reihe 12, Nr.490 (2002)

„24.Internationales Wiener Motorensymposium", VDI-Fortschritt Berichte, Reihe 12, Nr.539 (2003)

„25.Internationales Wiener Motorensymposium", VDI-Fortschritt Berichte, Reihe 12, Nr.566 (2004)

„26.Internationales Wiener Motorensymposium“, VDI-Fortschritt Berichte, Reihe 12, Nr.595 (2005)

„27.Internationales Wiener Motorensymposium“, VDI-Fortschritt Berichte, Reihe 12, Nr.622 (2006)

„28.Internationales Wiener Motorensymposium“, VDI-Fortschritt Berichte, Reihe 12, Nr.639 (2007)

„29.Internationales Wiener Motorensymposium“, VDI-Fortschritt Berichte, Reihe 12, Nr.672 (2008)

„30.Internationales Wiener Motorensymposium“, VDI-Fortschritt Berichte, Reihe 12, Nr.697 (2009)

„31.Internationales Wiener Motorensymposium", VDI-Fortschritt Berichte, Reihe 12, Nr.716 (2010)

„32.Internationales Wiener Motorensymposium“, VDI-Fortschritt Berichte, Reihe 12, Nr.735 (2011)

„33.Internationales Wiener Motorensymposium", VDI-Fortschritt Berichte, Reihe 12, Nr.749 (2012)

„34.Internationales Wiener Motorensymposium", VDI-Fortschritt Berichte, Reihe 12, Nr.764 (2013)

„35.Internationales Wiener Motorensymposium", VDI-Fortschritt Berichte, Reihe 12, Nr.777 (2014)

„36.Internationales Wiener Motorensymposium“, VDI-Fortschritt Berichte, Reihe 12, Nr.783 (2015)

„37.Internationales Wiener Motorensymposium“, VDI-Fortschritt Berichte, Reihe 12, Nr.799 (2016)

„38.Internationales Wiener Motorensymposium“, VDI-Fortschritt Berichte, Reihe 12, Nr.802 (2017)

„39.Internationales Wiener Motorensymposium“, VDI-Fortschritt Berichte, Reihe 12, Nr.807 (2018) 
Andreas Renschler,

Mitglied des Konzernvorstands der Volkswagen AG,

CEO Volkswagen Truck \& Bus $\mathrm{GmbH}$,

Volkswagen AG, Wolfsburg:

Wie Volkswagen Truck \& Bus die Zukunft des Transports mitgestaltet

Volkswagen Truck \& Bus and the Future of Transportation

Extraheft

Prof. KR Ing. Siegfried Wolf,

Verwaltungsratsvorsitzender,

Russian Machines LLC, Moskau:

Russland, ein schlafender Riese?

Russia - a Sleeping Giant?

Extraheft

Prof. Dr. Helmut List,

Vorsitzender der Geschäftsführung,

AVL List GmbH, Graz:

Antriebssysteme im Wandel

Propulsion Systems in Transition

Extraheft

Ing. F. Steinparzer, Dr. P. Nefischer, Dipl.-Ing. W. Stütz,

Dipl.-Ing. D. Hiemesch, BMW Motoren GmbH, Steyr:

Die Technik des neuen BMW Sechszylinder-Dieselmotors im X4 M40d.

The Technology of the New BMW 6-Cylinder Diesel Engine for the X4 M40d. 1 - 15

Dr.-Ing. A. Kufferath, Dr.-Ing. M. Krüger, Dipl.-Ing. D. Naber,

Dipl.-Ing. E. Mailänder, Dr.-Ing. R. Maier, Robert Bosch GmbH, Stuttgart:

Der Diesel Powertrain auf dem Weg zu einem vernachlässigbaren

Beitrag bei den $\mathrm{NO}_{2}$-Immissionen in den Städten

The Path to a Negligible $\mathrm{NO}_{2}$ Immission Contribution from the

Diesel Powertrain

Dr.-Ing. F.-T. Metzner, Dipl.-Ing. S. Willmann, Dipl.-Ing. C. Helbing,

Dr.-Ing. C. Eiglmeier, Dipl.-Ing. M. Köhne, Dipl.-Ing. A. Krause,

Dipl.-Ing. B. Wietholt, Volkswagen AG, Wolfsburg:

Die neue Vierzylinder-Dieselmotorengeneration von Volkswagen

The New Generation of Four-Cylinder Diesel Engines from Volkswagen

Dr. M. Kemmner, Ing. M. Mürwald, Dipl.-Ing. (FH) H. Sass,

Dipl.-Ing. M. Bertelmann, Dipl.-Ing. O. Erlenmayer, Dipl.-Ing. J. Larbi,

Dipl.-Ing. J. Böhm, Daimler AG, Stuttgart / Sindelfingen:

OM 654 1.6I - Der verbrauchsoptimierte 4-Zylinder-Einstiegsmotor

aus der neuen Dieselmotorenfamilie von Mercedes-Benz

OM 654 1.6I - The 4-Cylinder Entry Type of the

New Diesel Engine Family with Improved Fuel Efficiency

Extraheft

Dipl.-Ing. Dr. techn. C. Landerl, Dr.-Ing. J. Hahn, Ing. D. Kügler,

Dr.-Ing. B. Durst, Dipl.-Ing. S. Missy, BMW Group, München:

Der neue BMW 8-Zylinder Ottomotor.

The New BMW 8-Cylinder Petrol Engine. 
Dipl.-Ing. J. Königstedt, Dr. N. Ardey, Dipl.-Ing. A. Fröhlich,

Dipl.-Ing. J. Helbig, Dipl.-Ing. T. Reuss, Dipl.-Ing. J. Stotz,

AUDI AG, Neckarsulm / Ingolstadt:

Die konsequente Weiterentwicklung des neuen V6-TFSI-Motors von Audi der nächste Meilenstein der TFSI-Technologie

The Continued Development of the New Audi V6 TFSI Engine -

The Next Milestone in TFSI Technology

Dr. W. Demmelbauer-Ebner, Dr. J. Theobald, Dipl.-Ing. J. Worm,

Dr. P. Scheller, Volkswagen AG, Wolfsburg:

Der neue 1,5I EA211 TGI evo - Volkswagen gibt e-gas

The New 1.5I EA211 TGI evo - Volkswagen Steps on the e-Gas

Dipl.-Ing. C. Weber, Dr.-Ing. U. Kramer, Dipl.-Ing. R. Friedfeldt,

Dr.-Ing. H. Ruhland, Dr.-Ing. F. Krämer, Ford-Werke GmbH, Köln:

Entwicklung eines speziell für den Betrieb mit Methan ausgelegten

Verbrennungsmotors

Development of a New Combustion Engine Dedicated to

Methane Operation

Dipl.-Ing. C. De Marino, Dipl.-Ing. G. Maiorana, Dipl.-Ing. P. Pallotti,

Dipl.-Ing. S. Quinto, Dipl.-Ing. D. Sacco,

FCA EMEA Powertrain Engineering, Turin:

Der Global 3 und 4-Zylinder-Turbo Motor:

Die neue FCA-Familie von kleinen High-Tech Benzinmotoren

The Global Small Engine 3 and 4 Cylinder Turbo:

The New FCA's Family of Small High-Tech Gasoline Engines

J. Y. Kim MSc, I. S. Ryu MSc, J. N. Kim PhD, D. H. Han PhD,

K.-P. Ha PhD, J. Y. Kong MSc, J. D. Rho BSc, I. J. Hwang BSc,

K. S. Choi MSc, Hyundai Kia Motor Group, Namyang, Korea:

Hyundai-Kia's SmartStream 1.6L DI-Turbo Motor

Hyundai-Kia's SmartStream 1.6L Turbo GDI Engine

Dr. J. Adolf, Dr. C. Balzer, Dr. A. Janssen, Dr. W. Warnecke,

Shell, Hamburg;

Dr. K. Gruenberg, Dr. M. Klokkenburg, et al., Shell NL;

Dr. A. Mehta, Dr. J. Powell, Shell US; J. Cadu, Shell UK:

Der Weg zu nachhaltigen Kraftstoffen als Basis für

Zero Emission Mobilität - hat PtX eine Chance?

The Route of Sustainable Fuels as the Basis for

Zero Emission Mobility - has PtX a Chance?

Extraheft

Prof. Dr. R. Schlögl, Fritz-Haber-Institut der

Max-Planck-Gesellschaft (FHI), Berlin; Max-Planck-Institut

für Chemische Energiekonversion (CEC), Mülheim a. d. Ruhr:

Erneuerbare Energien in der Mobilität: Das Potential

synthetischer Kraftstoffe auf der Basis von $\mathrm{CO}_{2}$

Renewable Energies in Mobility: The Potential

of Synthetic Fuels Based on $\mathrm{CO}_{2}$

$191-201$ 
Dr.-Ing. M. Härtl, A. Stadler MSc, S. Blochum MSc, D. Pélerin MSc,

Dipl.-Phys. T. Maier MSc, Dipl.-Phys. V. Berger, Prof. Dr.-Ing. G. Wachtmeister,

Technische Universität München; Dr.-Ing. P. Seidenspinner, Dr. T. Wilharm,

ASG Analytik Service $\mathrm{GmbH}$, Neusäß;

Dr. E. Jacob, Emissionskonzepte Motoren, Krailling:

DMC+ als partikelfreier und potenziell nachhaltiger Kraftstoff für DI Ottomotoren

DMC+ as Particulate Free and Potentially Sustainable Fuel

for DI SI Engines

$202-229$

D. Neumann MSc, Prof. Dr.-Ing. S. Pischinger, M. Zubel MSc,

Dipl.-Wirt.-Ing. B. Heuser, K. Thenert MSc, Prof. Dr. rer. nat. W. Leitner,

RWTH Aachen University; Dr.-Ing. M. Schönen, Dr.-Ing. J. Schaub,

Dipl.-Ing. C. Jörg, FEV Europe $\mathrm{GmbH}$, Aachen:

Power-to-Liquids - Kompensation variierender E-Fuel

Zusammensetzungen mittels Digital Rate Shaping

Power-to-Liquids - Compensation of Varying E-Fuel

Compositions via Digital Rate Shaping

$230-258$

Dr. N. Steininger, Dr. D. Savvidis, Europäische Kommission, Brüssel:

$\mathrm{CO}_{2}$ Emissionen von schweren Nutzfahrzeugen in der EU:

Strategien und regulatorische Maßnahmen

$\mathrm{CO}_{2}$ Emissions from Heavy Duty Vehicles in the EU:

Strategy \& Regulatory Steps

Extraheft

Dr.-Ing. S. Klett, A. Haag MEng, Dipl.-Ing. (FH) P. Eder,

ZIEHL-ABEGG Automotive GmbH \& Co. KG, Kupferzell;

Dipl.-Ing. (FH) A. Angermaier, B. Langer BEng,

AVL Software and Functions $\mathrm{GmbH}$, Regensburg:

Hocheffizienter elektrischer Antrieb für Nutzfahrzeuge

High Performance Electric Drivetrain for Heavy Duty Vehicles

$259-272$

Dipl.-Ing. M. Bahne, U. Frey, GETRAG B.V. \& Co. KG, Magna Powertrain, Untergruppenbach / Köln; Dr. G. Schlager, Magna Powertrain, St. Valentin:

Beitrag von Getriebetechnologien zur $\mathrm{CO}_{2}$-Reduzierung -

Benchmark und Technologieausblick

Transmission Technology Contribution to $\mathrm{CO}_{2}$ Roadmap - A Benchmark

$273-290$

Dipl.-Ing. M. Sens, Dr.-Ing. E. Binder, Dipl.-Ing. A. Benz,

Dr. rer. nat. L. Krämer, Dipl.-Ing. K. Blumenröder,

Dipl.-Ing. M. Schultalbers, IAV GmbH, Berlin / Gifhorn:

Vorkammerzündung als Schlüsseltechnologie für

hocheffiziente Ottomotoren - neue Ansätze und Betriebsstrategien

Pre-Chamber Ignition as a Key Technology for Highly Efficient

SI Engines - New Approaches and Operation Strategies

Dr. E. Achleitner, Dipl.-Ing. H. Frenzel, Dipl.-Ing. J. Grimm, Dr. O. Maiwald, Dr. G. Rösel, Dipl.-Ing. P. Senft, Dr. H. Zhang, Continental, Regensburg:

Systemansatz für ein Fahrzeug mit Benzindirekteinspritzung und Partikel Filter im RDE

System Approach for a Vehicle with Gasoline Direct Injection and Particulate Filter for RDE

M. Younkins PhD, J. Serrano PhD, J. Fuerst BSME MBA,

S. Carlson BSME, Tula Technology, San Jose, USA;

A. Rayl MSEE, GM Global Propulsion Systems, Milford, USA:

Dynamic Skip Fire: Technologie für innovative Antriebe

Dynamic Skip Fire: Technologies for Innovative Powertrains

Extraheft 